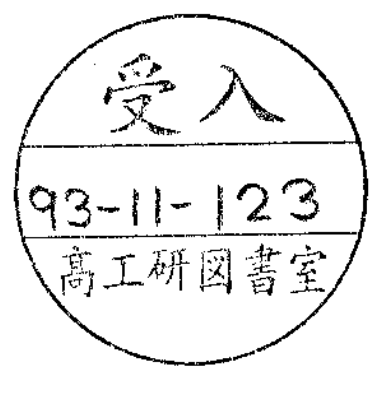

\title{
DESIGN AND PERFORMANCE OF AN ANALOG DELAY AND BUFFER CHIP FOR USE WITH SILICON STRIP DETECTORS AT LHC
}

\author{
The RD20 Collaboration
}

R. Brenner ${ }^{1}$, H. von der Lippe ${ }^{2}$, J. Michel ${ }^{3}$, E.Nygård $^{4}$, T. Ødegaard 5 , N.A.Smith ${ }^{6}$, P.Weilhammer ${ }^{1}$, K. Yoshioka ${ }^{1}$

\begin{abstract}
An Analog Delay and Buffer Chip has been designed and built in $1.2 \mu \mathrm{m}$ CMOS technology to be used in Silicon Detectors at LHC. Measurements on the performance of the prototype chip are presented. The storage cells variations are smaller than $0.65 \mathrm{rmsV}$, i.e. $1 / 100$ of the signal in its input for a minimum ionizing particle.
\end{abstract}

Submitted to Nuclear Instruments and Methods A

1) CERN, Geneva, Switzerland

2) Senter for Industriforskning, Oslo, Norway. Now at Lawrence Berkeley Laboratory, Berkeley CA, USA

3) LEPSI, Strasbourg, France

4) Senter for Industriforskning, Oslo, Norway. Now at Oslo University, Oslo, Norway

5) Senter for Industriforskning, Oslo, Norway.

6) Liverpool University, Liverpool, UK 


\section{INTRODUCTION}

Silicon microstrip detectors have a number of features to make them promising candidates for use in proposed experiments at the future Large Hadron Colliders. They are fast, with signal collection speeds in the range 20 to $30 \mathrm{nsec}$, relatively radiation hard and can be built with very high granularity well matched to the high particle density expected in $T e V$ hadron collisions.

The requirements on the associated front-end electronics to read out minimum ionising particle signals from interactions at LHC are very demanding. The following parameters have been used as preliminary guidelines for the data handling capability required by the front-end electronics:

interval between each beam crossing

first level trigger delay

first level trigger frequency

second level trigger delay

second level trigger frequency

$\begin{array}{ll}: & 15 \mathrm{nsec} \\ : & 1 \mu \mathrm{sec} \\ : & 100 \mathrm{kHz} \\ : & 20-100 \mathrm{msec} \\ : & 1 \mathrm{kHz}\end{array}$

Several approaches have been followed to implement front-end electronics which can fulfil all the requirements of future experiments at LHC and SSC, in particular for tracking devices very near the interaction region [1. Within the RD20 collaboration [2] a novel front-end concept has been proposed [3]. In this approach, the emphasis is put on a full analog low noise and low power readout implemented throughout in CMOS.

A fast preamplifier circuit is combined with a moderately fast shaper circuit in order to achieve full charge collection with optimal noise performance and acceptable power consumption. That input stage is followed by an analog delay and buffer unit to preserve the information of a particular event until the arrival of a first level trigger signal.

Allowing a shaping time of signals long compared to the bunch crossing time requires a mean to retrieve exact timing information. This is achieved by deconvoluting the sampled pulse shape with a relatively simple analogue deconvoluting circuit (APSP) [4], which reconstructs the original time interval in which the charge pulse occupied at the detector.

In this paper a description of the design and the performance of the Analog Delay Buffer chip used in this architecture is presented. 


\section{THE RD20 FRONT-END ELECTRONICS CONCEPT}

The basic design concept proposed by RD20 for the front-end electronics for a silicon tracker at the LHC is outlined in Fig. 1.

The signal from the detector is amplified with a charge sensitive preamplifier followed by a CR-RC shaper with a peaking time of $45 \mathrm{~ns}$. The shaped signal is continuously sampled in the Analog Delay and Buffer (ADB) with a sampling frequency of 67.7 MHz. Inside the ADB, the samples are delayed by $1 \mu \mathrm{sec}$, which is the assumed time for the Level 1 Trigger decision to be taken. Whenever a positive Level 1 Trigger decision arrives, 4 samples associated with that trigger are tagged and protected from overwriting until they transferred to the Analog Pulse Shape Processor (APSP). Storage cells containing samples having a delay of more than $1 \mu \mathrm{sec}$ and not having a tag are overwritten with new information.

In the APSP, a deconvolution filtering operation takes place by utilising 3 of the transferred samples to restore the original signal from the strip detector (the 4th sample is presently not being used). The deconvolution principle has been described in [5].

The modules of the proposed system shown in Fig. 1, i.e. the amplifier \& shaper, the ADB and the APSP have all been designed, produced and tested in prototype form. Presently they are physically located on three separate chips, but their integration into one single chip is under way.

The object of this paper is to present the functionality of the ADB and the results from the evaluation of it.

\section{THE ANALOG DELAY AND BUFFER}

The ADB consists of an analog section and one digital control-logic section. The analogue part consists of 4 identical, parallel channels each featuring an input amplifier followed by 84 storage cells and thereafter an output amplifier. In Fig. 2, these basic elements are outlined.

The control-logic controls the flow of data in the analogue section by, for instance, providing the sampling clock of the input signal, controlling the delay of the samples, controlling the buffering of triggered samples prior to the APSP, and controlling the delivery of the samples to the APSP at the right time. The control-logic is subdivided into a Control Unit and Registers which contains pointers; the main Registers are illustrated in Fig. 3. 
The input signal is continuously sampled every 15 nsec. Each sample is stored in the analogue cell which at a given moment is addressed by the write pointer. Once the write pointer reaches the last available position of the storage cell's array, it jumps back again to the first available position. The trigger 1 pointer is running behind the write pointer spaced by $1 \mu \mathrm{sec}$ which is the anticipated delay of the $\mathrm{T} 1$.

Whenever a positive $\mathrm{T} 1$ decision arrives, 4 cells associated with this event are turned into a temporary 4 cell buffer-zone which is protected from being overwritten until it has been read out and hence released. Three out of the 4 cells contain the necessary information for performing the deconvolution and the fourth cell holds the analogue value of the peak of the shaped pulse. Each of the individual cells in a buffer-zone are marked in the trigger-tag register, which stores every buffered cell regardless which buffer-zone it belongs to, and in one of the event registers, which stores only the group of 4 cells belonging to one buffer-zone. The cell which contains the first sample in a buffer-zone is marked in the first sample register.

The readout of the events (buffer-zones) is sequential, i.e. first event in becomes the first event out. This is organised in such a way that whenever a new readout cycle starts, the event register which contains the buffer-zone which now is the oldest will be found and selected. In order to find the first cell in the buffer-zone to be read out, the Control Unit looks in the first-sample register. In this register there will be one and only one tag which has a corresponding position to one of the 4 tags in this event register. The cell which corresponds to this coincidence will then be read out first and the other 3 will follow in sequence. After the readout, the buffer-zone will be released by removing the tags in the trigger-tag register.

The writing of new samples is unaffected by the readout and hence the circuit is able to write into a new cell every $15 \mathrm{~ns}$. This means that the write pointer will frequently pass by cells which it cannot write into because the cell has turned into a buffer-zone cell which has not yet been read out. Whenever this situation occurs, a mechanism is implemented which will force the write pointer to skip all cells of this kind and jump until it finds a free cell. This mechanism is called "bypass". It utilises the trigger-tag register for this purpose, which as already described contains all the cells which are tagged. The "bypass" mechanism is illustrated in Fig. 4.

In the present design there is an upper limit of 4 dynamically located T1 buffer-zones in the ADB which can be used simultaneously (each buffer-zone represented by 4 samples). Since the readout of the ADB runs at approximately $4 \mu \mathrm{sec} /$ buffer-zone (the readout speed is determined by the processing time needed by the APSP) and a new buffer 
is released after each readout, the probability of an overflow is small [6] since the average Level 1 trigger rate is only $100 \mathrm{kHz}$.

The ADB prototype circuit has been implemented in a $1.2 \mu \mathrm{m}$ CMOS technology with a total power consumption of approximately $50 \mathrm{~mW}$, which for a 128 channel full version chip would scale to approximately $0.4 \mathrm{~mW} /$ channel since the present 4 analogue channels can be extended to 128 without any other changes. The input amplifier and the output amplifier shown in Fig. 2 are not foreseen in a final integrated version of the system. They are incorporated in this prototype only in order to permit the stand-alone tests.

\section{MEASUREMENTS}

\subsection{Set-Up}

For bench test purpose, the electronic environment in which the ADB will operate at LHC was emulated by signals from pulse generators: BCO clock, READ clock, Level 1 Trigger (T1), IDLE . Figure 5 shows the measurement set up.

The Level 1 Trigger is used to tag samples read into the ADB $1 \mu \mathrm{sec}$ before. The T1tagged samples are retrieved serially on the falling edge of the IDLE signal. The readout speed is determined by the READ clock, which in these tests ran at $1 \mathrm{MHz}$.

\subsection{Signal Transmission}

In order to show that the ADB's delay function is correct, one period of a sine wave was put into the ADB and was retrieved delayed by $1 \mu \mathrm{sec}$. Each sample can be retrieved in any of the four periods per event readout cycle. Then, one of those periods can be used to scan the delayed signal by keeping the sine wave fixed in relation to the reset (to make sure that the samples from the signal are stored in the same storage cells) and moving the $\mathrm{T} 1$ to get the samples on the desired period. Figure 6 shows the signal applied to the input of the ADB and the corresponding signal on the 2 nd and 4 th periods of the output.

The amplitude of the output voltage of the shaper corresponded to the charge created by 0.5 Minimum Ionizing Particle at the input.

\subsection{Storage Cell Variations}

To verify the magnitude of the variation amongst storage cells due to the processing of the chip or due to pick ups from switching circuitry, three different DC levels were applied to the input of the ADB and, for each dc level, the output signal from all the 84 storage cells measured. The reason for this approach is that the signal is written onto the 
capacitor as a voltage but it is the corresponding charge which is read out again (although transformed into voltage once more before sent out from the chip). Hence, if there were variations in the capacitor values, the variations on the output depend on the input $\mathrm{dc}$ voltage level.

An example of those measurements is shown in Fig. 7, and it shows in addition to variations between cells that there is a small $\mathrm{dc}$ level shift between the 1st and the last cell. The variation of the dc output level along the storage cells is $0.75 \mathrm{mV}$ but it is independent of the dc input level. A linear fit was done to the curve in Fig. 7 and the r.m.s variations of the residues are listed in Table 1.

The results show that the non-uniformity among storage cells is not correlated with the input level and variations from cell to cell are in all cases smaller than $2 \mathrm{mV}$ with r.m.s. values smaller than $0.65 \mathrm{mV}$. This indicates that the variation are not due to a spread in the capacitance of individual cells but probably caused by variations of charge injection from switches along the storage cell's array.

\subsection{Bypass Test}

In order to test the bypass control circuitry, a sine wave and a triangular wave were fed into the ADB as shown in Fig. 8.

The set-up was made such that the externally applied T1 pulses for the two different waveforms were displaced by $82 \mathrm{BCO}$ clock-periods. In this way, the buffer-zone created due to the first T1 will force the buffer-zone created due to the second T1 to be split in two halves, the first two cells in front of first buffer-zone and the last two following behind (overall buffer length is 84 cells).

Reading out these two events ( 8 cells in total), the sine and the triangular waves can be reconstructed by repeating the procedure used in 4.2 , now using the 1 st and 5 th read out periods (as well 2 nd and 6th, 3rd and 7 th or 4 th and 8 th periods) for scanning the delayed signals. This is shown in Fig. 8. It shows that the cell-skipping mechanism works correctly.

\section{CONCLUSIONS}

From the tests on the Analog Delay and Buffer described in this paper, one can conclude that the basic functionality of the device is correct. Signals are transmitted with small distortion in comparison with the overall noise in the system, and the variations between storage cells are less than $1 \mathrm{mV}$ r.m.s, which is well below the foreseen noise performance of the circuit. 


\section{Acknowledgements}

This work has been funded by CERN and several national agencies:

IN2P3 (France), NAVF (Norway), SERC (UK). We should like to thank them all for their support. 


\section{REFERENCES}

[1] SDC Silicon Tracker Conceptual Design Report (1992).

[2] H. Borner et al., The Development of High Resolution Silicon Strip Detectors for Experiments at High Luminosity at the LHC, CERN/DRDC 91-10 (1991).

[3] RD20 Status Report, CERN/DRDC 92-28, May 1992.

[4] N. Bingefors et al, A Novel Technique for Fast Pulse-Shaping Using a Slow Amplifier at LHC, Nuclear Instruments and Methods A326 (1993) 112.

[5] S. Gadomski et al., The Deconvolution Method of Fast Pulse Shaping at Hadron Colliders, Nuclear Instruments and Methods A320 (1992) 217.

[6] G. Hall, RD20 Pipeline Buffer Depth and Inefficiency, RD20/TN/12, Jan 1993. 
Table 1

\begin{tabular}{|c|c|}
\hline$V_{\text {in }}(\mathrm{mV})$ & $\sigma(\mathrm{mV})$ \\
\hline \hline 0.0 & 0.61 \\
\hline 10.0 & 0.63 \\
\hline 20.0 & 0.49 \\
\hline
\end{tabular}




\section{Figure Cantions}

Fig. 1 : RD20 Front-End Electronics. The Analog Delay and Buffer (ADB) is continuously sampling the output of the Amplifier. When a positive Level 1 Trigger arrives, 4 samples are tagged. Eventually, the tagged samples are processed by the APSP.

Fig. 2 : Analog chain of the ADB. The input and output amplifiers are present only in the prototype chip to permit the stand-alone tests.

Fig. 3 : Main Registers and Pointers. The Trigger1 pointer is running behind the Write pointer by $1 \mu \mathrm{sec}$. Whenever the Level 1 Trigger arrives, the storage cells pointed by the Triggerl are tagged in the Trigger Tag register creating one buffer zone, and the first sample of that event are tagged in the 1st Sample register.

Fig. 4 : Bypass mechanism. The samples from the Amplifier output are written continuously but not necessarily in contiguous storage cells.

Fig. 5 : Schematic of the set-up for testing the ADB.

Fig. 6 : Signal transmission :

(a) Input. The amplitude of the sine wave corresponds to the peak value of the output of the Amplifier for a 0.5 minimum ionizing particle. The sinus wave is continuously sampled by the BCO clock.

(b) Output. The sampled values of the sinus wave are delayed and shifted out of the ADB by moving the Trigger Level 1 (T1).

Fig. 7 : Storage cell variation.

Fig. 8. : Bypass :

(a) Input. The sinus wave and the triangular wave are spaced by $82 \mathrm{BCO}$ clocks. A Trigger Level 1 (T1) is arrives creating a buffer-zone with samples from the sinus wave. Another Trigger Level 1 is sent creating another bufferzone with samples from the triangular wave. The spacing of these waves ensures that the samples from the triangular wave are not written in contiguous storage cells.

(b) Output. The 3rd sample from the 1st $\mathrm{T} 1$ and the 3rd sample from the 2 nd $\mathrm{T} 1$ are in the same picture, showing that the bypass circuitry is working correctly. 


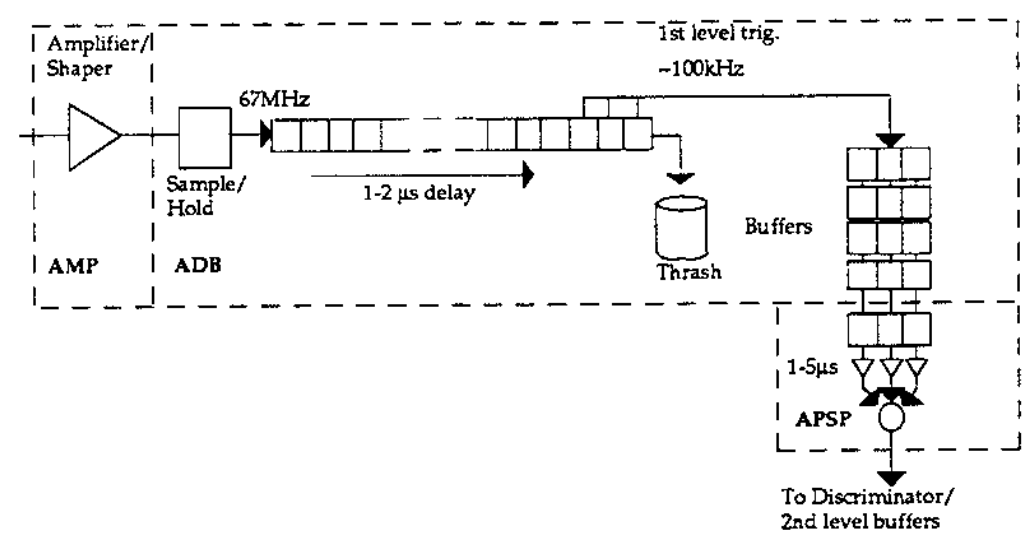

Fig. 1 


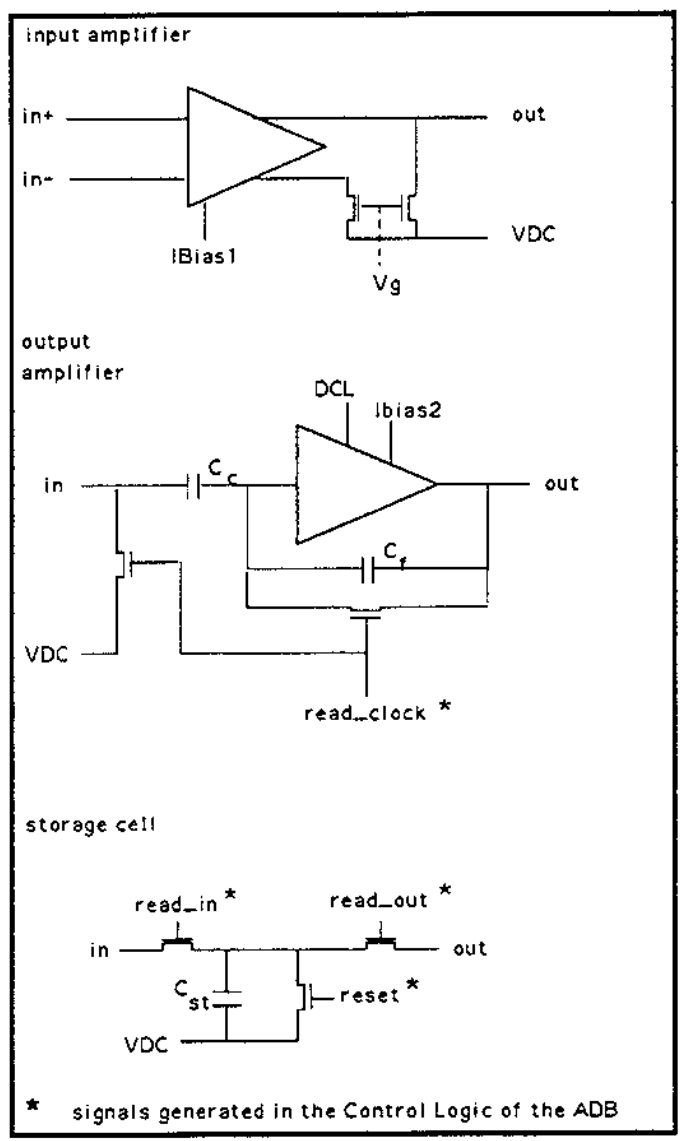

Fig. 2 
Storage Cell's Array

\begin{tabular}{l}
1 \\
$\mid w w w w x$ \\
\hline
\end{tabular}

$\uparrow$

Trigger1 $\uparrow$

Write

Trigger Tag Register

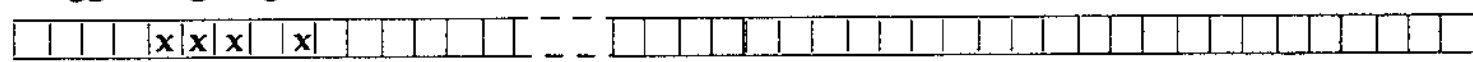

1st Sample Register

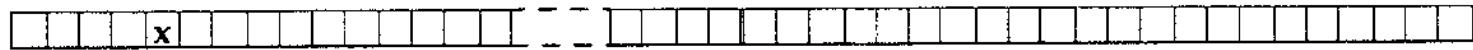

Event Registers

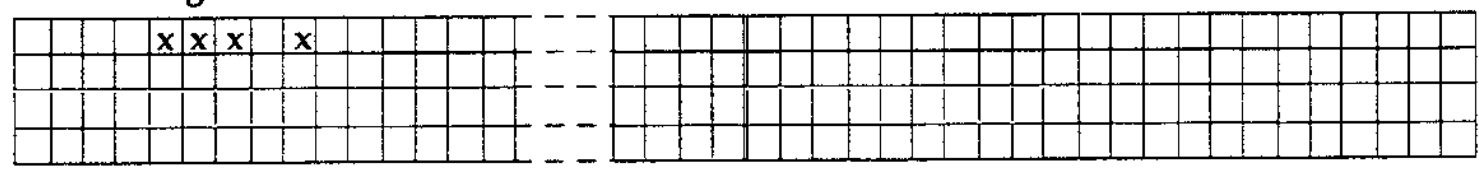

Fig. 3 
Storage Cell's Array

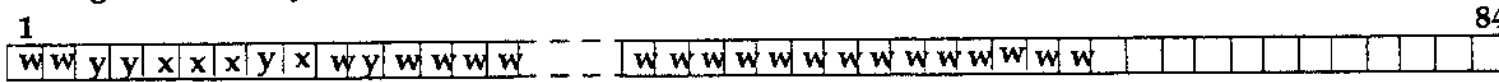
$\uparrow$

1

Trigger1

Write

Trigger Tag Register

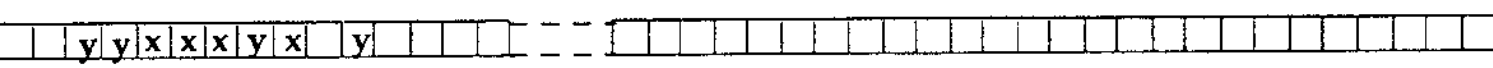
1st Sample Register

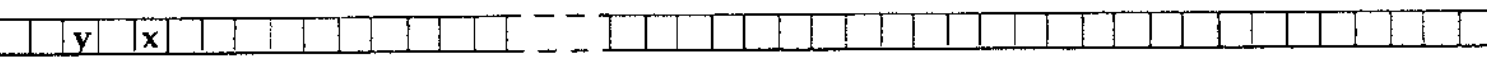

\section{Event Registers}

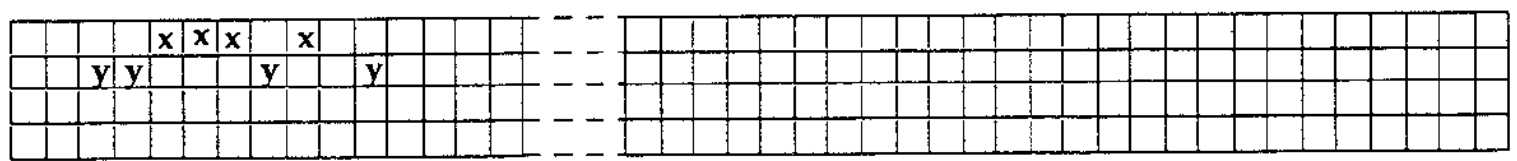

Fig. 4 


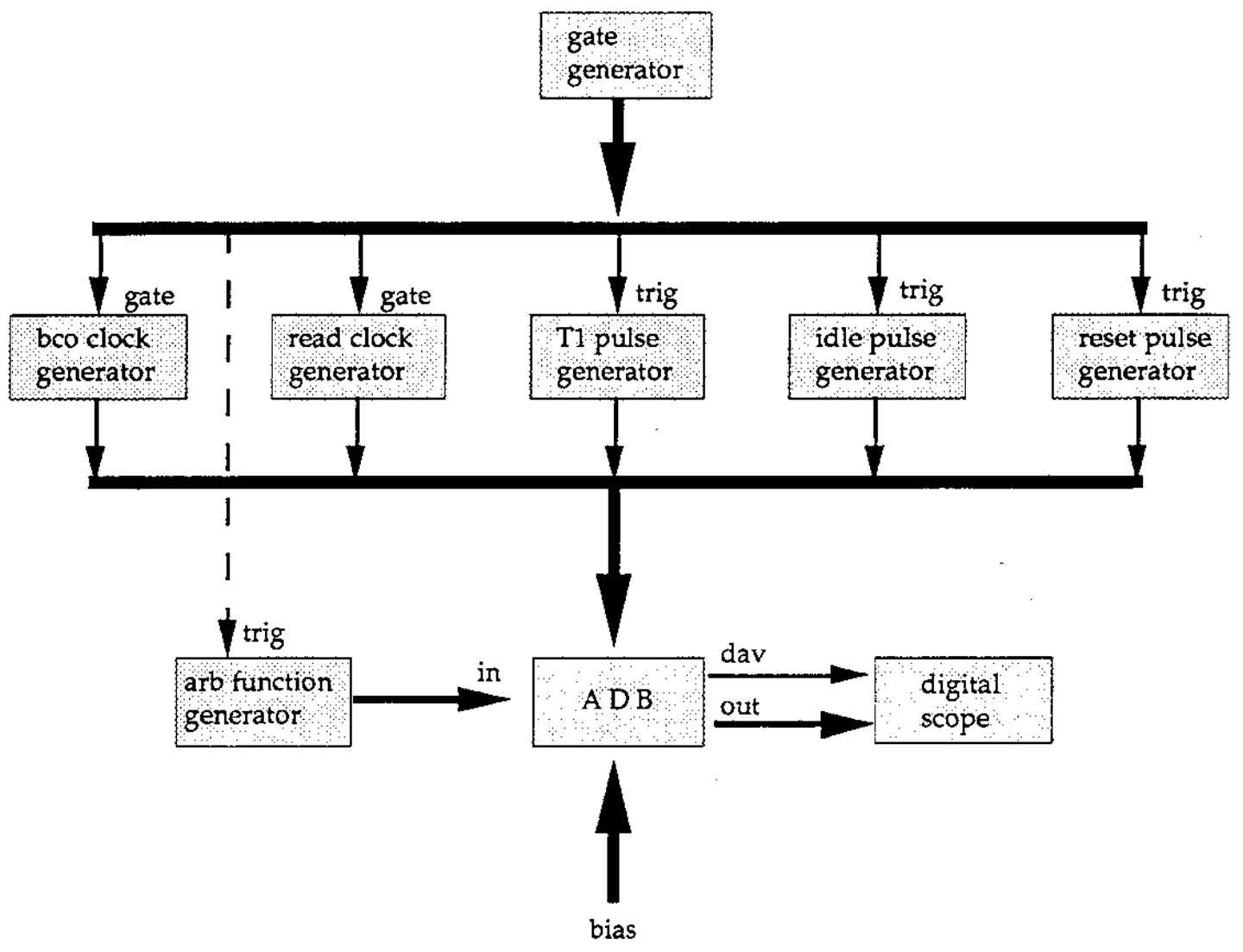

Fig. 5 

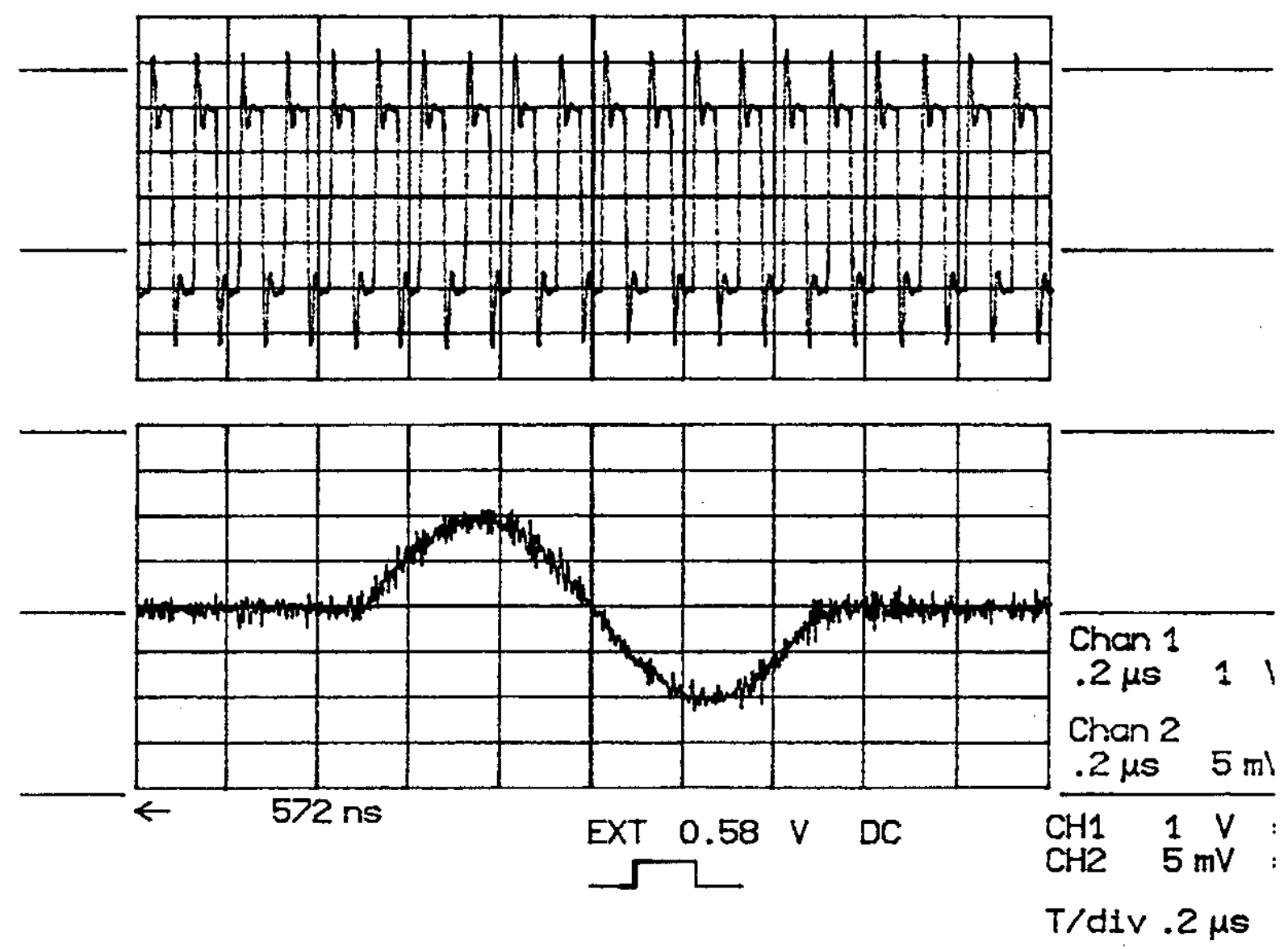

Fig. 6a 

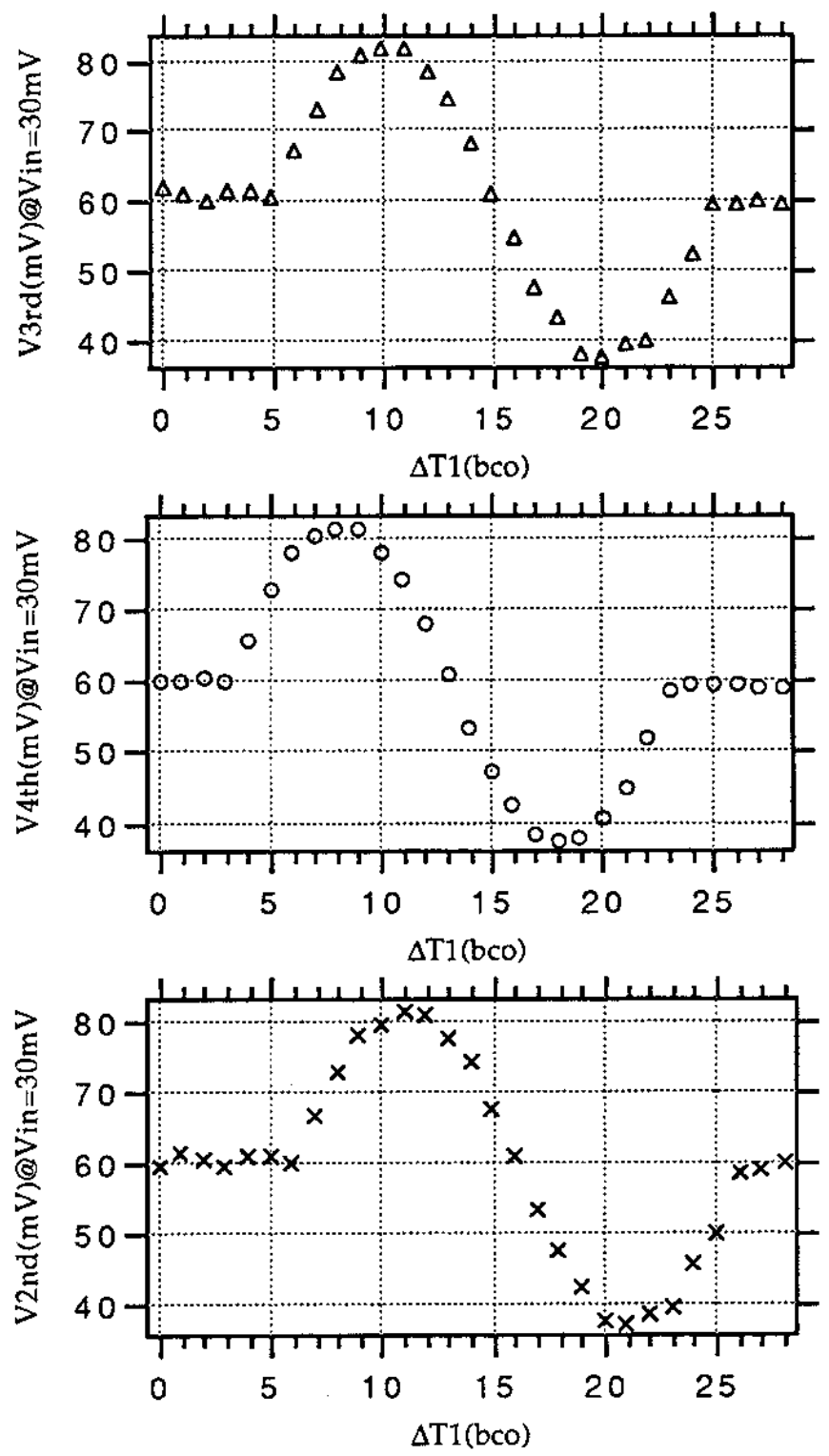

Fig. 6b 


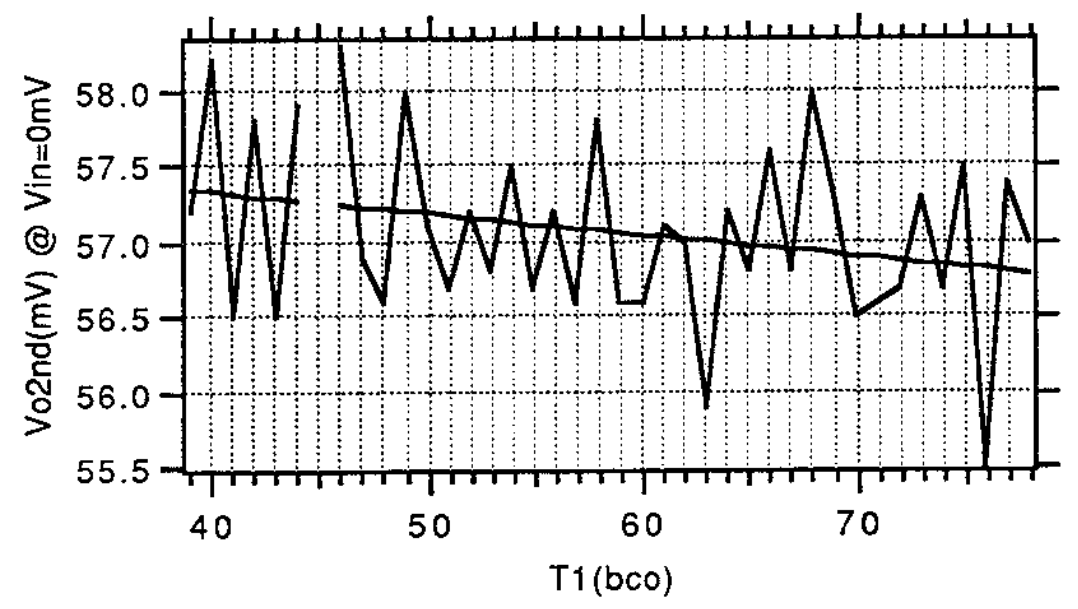

Fig. 7 

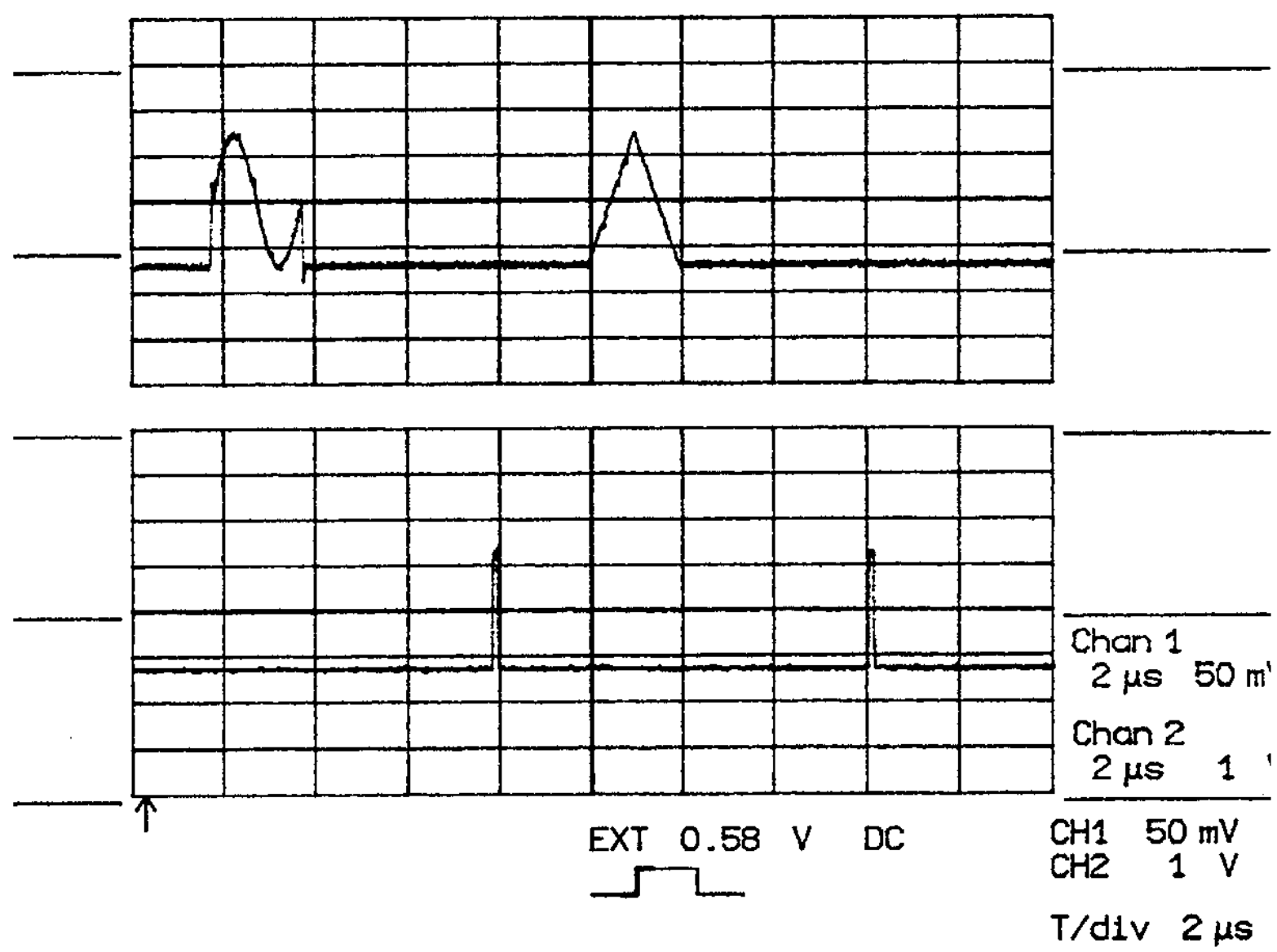

Fig. 8a 


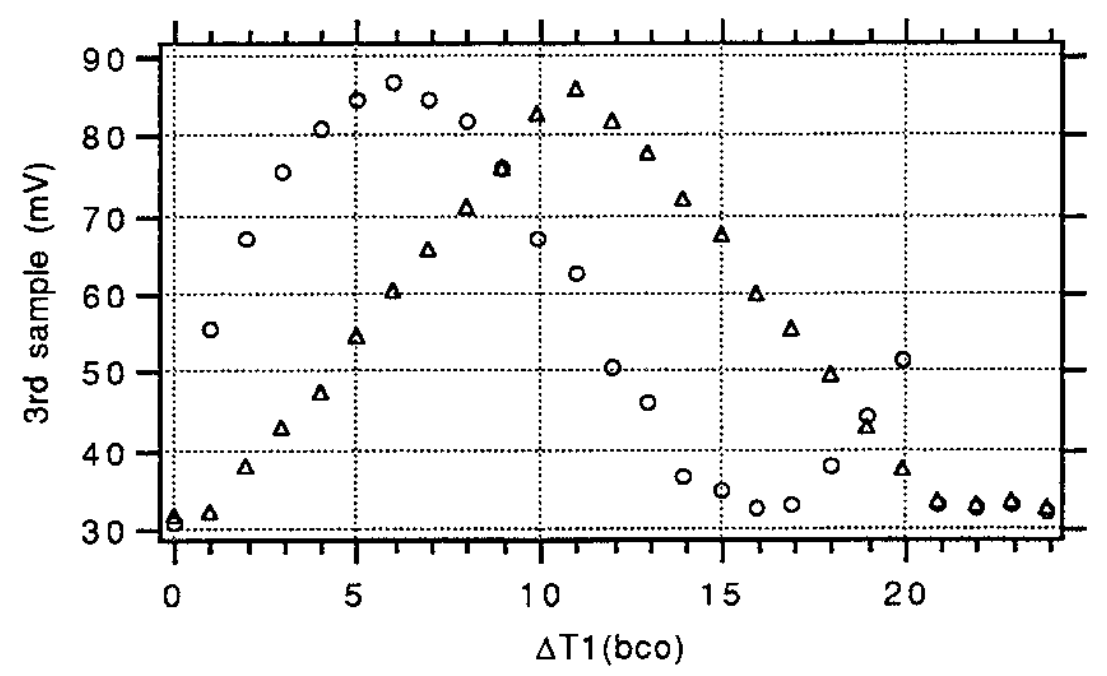

Fig. $8 \mathrm{~b}$ 